\title{
Arthroscopic Management of Type II Superior Labrum Anterior to Posterior (SLAP) Lesions: Minimum 2-Year Follow Up
}

\author{
Emad Abdelhadi ${ }^{1^{*}}$, Mohamed El Sadek ${ }^{2}$ \\ ${ }^{1}$ Assistant Professor of Orthopedic Surgery, Zagazig University, Egypt \\ ${ }^{2}$ Assistant Professor of Orthopedic Surgery, Zagazig University, Egypt
}

*Corresponding Author: Emad Abdelhadi, Assistant Professor of Orthopedic Surgery Department, Zagazig University, Egypt.

\begin{abstract}
Background: Superior labrum anterior to posterior (SLAP) lesions are tears that expand anteriorly from the posterior superior labrum, including the long head of the biceps tendon originating from the glenoid labrum, and halting before the anterior glenoid notch.
\end{abstract}

Aim of the work: Assessment of clinical outcome of the arthroscopic type II anchor repair of superior labrum anterior to posterior lesions (SLAP).

Patients and Methods: This prospective study of Twenty five patients diagnosed as type II superior labrum anterior to posterior (SLAP) lesion. All had been managed by arthroscopic anchors repair started May 2015 till September 2018 in our university hospital. The age ranging from 20 to 48 years wasmean35.23; three patients (12\%) females and 22 patients (88\%) were males. Type II SLAP lesions that did not respond to nonsurgical treatment and SLAP lesions either isolated or associated with other lesions (Bankart lesion \& Partial rotator cuff lesions) were Inclusion criteria. Shoulders with intra-articular chondral damage, full thickness rotator cuff tear, rotator cuff repair, labral repair outside the superior labrum, the bony subacromial decompression, and acromioclavicular joint resection were excluded.

Results: Males were 22 (88\%) patients and the females were $3(12 \%)$ patients. Their age was ranging from 20 to 48 years with the mean 35.23 years. All were unilaterally affected with 20 patients (80\%) dominant side and 5 patients (20\%) non-dominant side. athletes were eleven patients (44\%), six patients (24\%) Labors and Eight patients (32\%) workers. University of California at Los Angeles (UCLA) score after 12 months from operation was 33.24 with rang from 22-36. The UCLA score comparison pre-operation with the postoperative UCLA score at six months and one year showed highly significant improvement pre-operative, six months post-operative and the $P$ value after one year post-operation was $<0.001$. The relation between sex and UCLA score 6 month post-operative is insignificant ( $P$-value 0.87$)$ and one year ( $P$-value 0.39$)$.

Conclusion: Treatment of SLAP lesions by arthroscopy is challenging and provides good functional outcome.

Keywords: Biceps anchor $\bullet$ SLAP tear $\bullet$ SLAP repair $\bullet$ Biceps tenodesis $\bullet$ Shoulder arthroscopy.

\section{INTRODUCTION}

Andrews et al. (1) were the first to show that tears in the Superior glenoid labrum cause shoulder pain in a popularity tossing competitor (pitchers with a lot of experience). During the tossing motion, massive pressure was applied to the shoulder joint and the biceps brachialis muscle, resulting in the pathology. The goal of the study was to define the labrumpathology in overhead throwers. Snyder et al (2) coined the term Superior Labrum Anterior and Posterior (SLAP) tear.

The long head of the biceps tendon (which originates from the glenoid labrum) is stretched out from the posterior superior labrum and comes to a halt just before the anterior glenoid notch in SLAP lesions (1). Lesions of the superior glenoid labrum and biceps anchor are a common cause of shoulder pain in athletes (2). Advances in shoulder arthroscopy have aided in the understanding and treatment of superior labral anterior-posterior (SLAP) tears (3).

Most recent biomechanical considers have planned a few suggestions for the pathogenesis of SLAP tears in tossing competitors and the effect of these injuries on characteristic shoulder biomechanics (4). Progresses in imaging procedures obligate encouraged better accuracy 
in make a diagnosis SLAP tears. The investigation of clinically appropriate SLAP tears stays challenging as there are no specific assessment findings and the relapse of associated shoulder injuries (5).

Conclusive finding of supposed SLAP tears is established throughout arthroscopic assessment (6). Advancement in surgical procedures had made it promising to attain precise repair in selected types of injury. Modern studies have revealed surely respectable functional results and a satisfactory rate of return to sport and/or work with arthroscopic SLAP tears treatment (2).

\section{Patient And Method}

This prospective study contained of twenty five patients who had shoulder arthroscopy designed for the management of isolated type II SLAP injuries or associated with incomplete tear of rotator cuff or Bankert lesion between May 2015 and September 2018 in our university hospital. Patients mean age was 35.23 alternating from 20 to 48 years. Patients diagnosed thorough clinical consideration (history, clinical presentation and examination), radiological analysis (plain x-ray \& MRI), SLAP lesions that did not respond to nonsurgical treatment, and last of all SLAP lesions either isolated or concomitant with other lesions (Bankart lesion \& rotator cuff lesions). Shoulders with intra-articular chondral injury, complete rotator cuff tear, rotator cuff repair, labral repair outside of the superior labrum, bony subacromial decompression, and acromioclavicular joint resection were excluded.

\subsection{Surgical Procedures}

To anesthetize the patient, general anesthesia is used. The preliminary operative evaluation includes an anesthetic examination. At $0^{\circ}$ and $90^{\circ}$ forward elevation, the shoulder joint is examined for passive ROM, inferior sulcus translation in comparison to the contralateral side, and irregular posterior or anterior load and shift in the scapular plane (7). A standard posterior viewing entry and anterior rotator interval working entry are used to evaluate the glenohumeral joint. All of the articular surfaces, as well as the biceps tendon, glenoid labrum, and rotator cuff, should be probed and examined.

The superior labrum, biceps tendon, and biceps anchor are all checked for wear and instability. A probe can be used to move the superior labrum beyond the glenoid's articular cartilage border, exposing subchondral bone beneath, resulting in Type II SLAP or unstable tear. Debride the areas that will be worn. For completing the restoration of an unstable SLAP, portals are well-known. The Wilmington posterior-lateral portal is used in conjunction with the anterior-superior rotator interval portal (Fig. 1) (8). The neck of the glenoid and the rim are roughened with a shaver after debridement of labral wear. Suture anchors are implanted into the glenoid rim at a $45^{\circ}$ angle through the working portals. Depending on the degree of anterior and posterior superior labral detachment, suture anchors are placed on the glenoid at 10,11, 1, or 2 o'clock. The damage is usually repaired with one or two anchors.

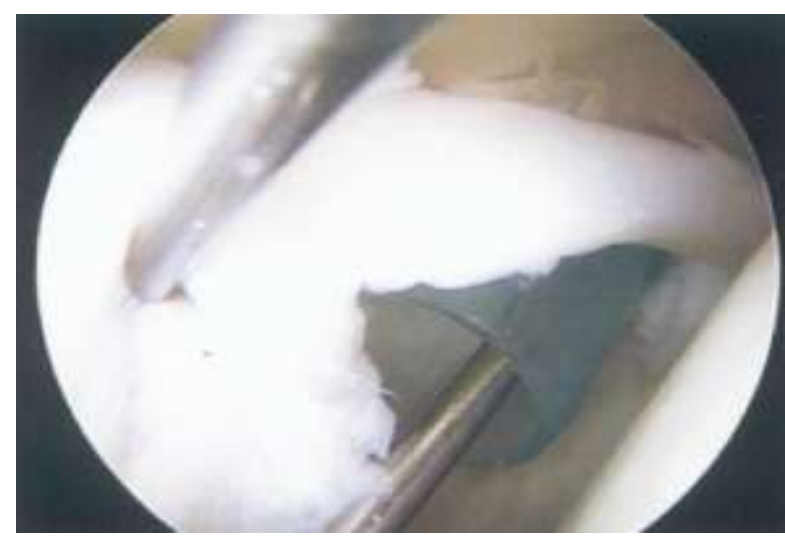

Figure1. Working Portal of Slap Repair

Using an arthroscopic penetrating instrument loaded with a shuttle suture or a lasso suture, a single vertical stitch from each suture anchor is located around the inner border of the labrum centrally and completes the capsulolabral tissue peripherally. The labrum is secured to the glenoid by a sliding arthroscopic knot reinforced with alternating half-hitches (Fig. 2). 


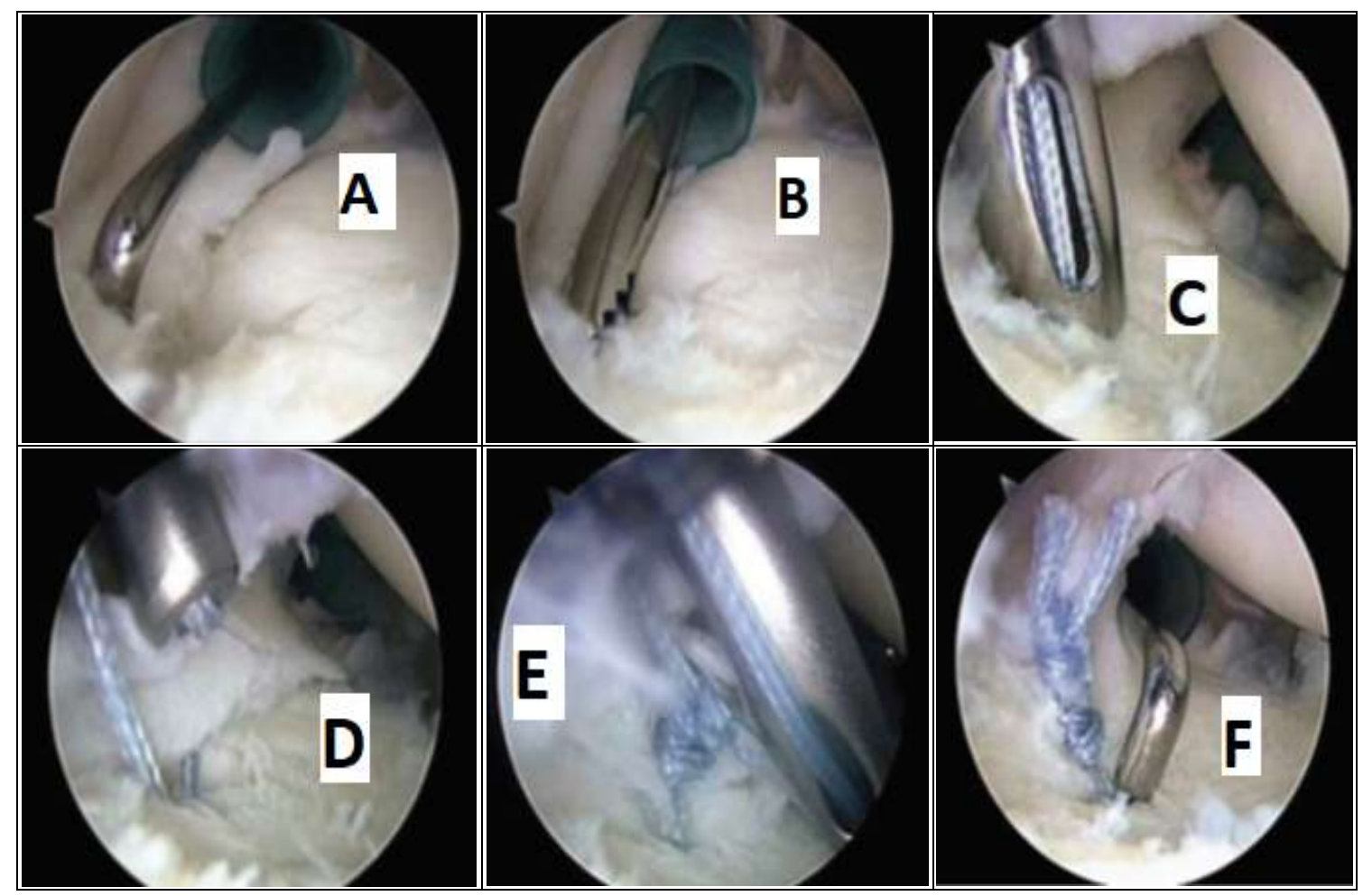

Figure2. Steps of SLAP lesion repair using suture anchor

Patients share in a comprehensive rehabilitative procedure after surgery, which is overseen by a physical therapist. During the first three to six weeks, sling immobilization with swing, elbow, and wrist exercises is started. Range-of-motion exercises, both active-assisted and passive, are started at three to six weeks. The rotator cuff has been strengthened to a higher level and scapular stabilizers begins after 6 weeks. Patients begin a vigorous exercise program three months after the operation. The patients were then observed every two months for the next six months, until one year after surgery, and the UCLA scores were calculated. Patients are allowed to return to full active work when they have regained $80 \%$ of their motion and strength, as well as the ability to meet the physical requirements of their job.

\section{Results}

We had twenty-two (88\%) of the patients were males with only three females (12\%). The mean age at the time of procedure was 35.23 extending from 20 to 48 years. All patients were unilaterally with the main side injured in 19 patients $(76 \%)$ and the non-main side in six patients $(24 \%)$. Eleven patients $(44 \%)$ were competitors, six patients (24\%) hard workers and eight patients (32\%) employees. (Table. 1)
Table1. Patients Data

\begin{tabular}{|l|l|l|l|}
\hline \multirow{2}{*}{ Age } & Mean & \multicolumn{2}{|l|}{35.23} \\
\cline { 2 - 4 } & Range & \multicolumn{2}{|l|}{$20-48$} \\
\hline \multirow{3}{*}{ Activity } & Male & 22 & $88 \%$ \\
\cline { 2 - 4 } & female & 3 & $12 \%$ \\
\hline \multirow{3}{*}{ Injury Side } & & No & $\%$ \\
\hline \multirow{2}{*}{ Dominant Hand } & Competitors & 11 & $44 \%$ \\
\cline { 2 - 4 } & Hard Workers & 6 & $24 \%$ \\
\cline { 2 - 4 } & Employees & 8 & $32 \%$ \\
\hline \multirow{2}{*}{ Mechanism Of Injury } & Right & 19 & $76 \%$ \\
\cline { 2 - 4 } & Left & 6 & $24 \%$ \\
\hline \multirow{2}{*}{ Slab Type } & Right & 20 & $80 \%$ \\
\cline { 2 - 4 } & Left & 5 & $20 \%$ \\
\hline & Trumatic & 10 & $40 \%$ \\
\cline { 2 - 4 } & Atrumatic & 15 & $60 \%$ \\
\hline & Type 2 & 19 & $76 \%$ \\
\hline & TYPE 5 & 6 & $24 \%$ \\
\hline
\end{tabular}

Ten patients (40\%) had an isolated SLAP lesion while the lasting $15(60 \%)$ had concomitant lesions; 8 had concomitant bankart lesion, 7 had subacromial impingement and one with a small Avulsion of the Articular Side Tendon (PASTA) lesion. The anchor centrally positioned in nine patients $(36 \%)$ and non-central anchor position in 16 patients (64\%). One anchor fixation in twenty patients $(80 \%)$ and two anchors in five patients $(20 \%)$.

All patients were evaluated pre and postoperatively at six months and one year using the 
UCLA score. Preoperative UCLA score was 18.23 with range from 15 to 22 . Post-operative UCLA score at six months was 29.65 with range from 16 to 36 . UCLA score after 12 month's post-operation was 32.24 with rang from 22 to 36. (Table. 2). Comparing UCLA score preoperation with the UCLA score at six months and at one year post-operative displayed highly significant improvement ( $p$ more than 0.001).

Table2. UCLA score before operation and 6 months after and 12 months after operation

\begin{tabular}{|l|l|l|}
\hline \multicolumn{2}{|l}{} & $\mathrm{NO}=25$ \\
\hline \multirow{2}{*}{ UCLA Score Before Operation } & Mean & 18.23 \\
\cline { 2 - 3 } & Range & $15-22$ \\
\hline \multirow{2}{*}{ UCLA 6 Month After Surgery } & Mean & 29.65 \\
\cline { 2 - 3 } & Range & $16-35$ \\
\hline \multirow{2}{*}{$\begin{array}{l}\text { UCLA Score 12 Month After } \\
\text { Surgery }\end{array}$} & Mean & 33.24 \\
\cline { 2 - 3 } & Range & $22-36$ \\
\hline
\end{tabular}

There is insignificant relation among sex and UCLA score at six month and one year (P
$>0.05$ ). The left dominant had statistically significant better clinical results than nondominant at six months, one year and final follow up $(\mathrm{P}$-value $<0.05)$. There was statistically insignificant difference of the clinical results post-operatively at six months and one year (P- value above 0.05). Although the non-traumatic patients had better UCLA score, these were statistically insignificant at six month and one year and at final follow up (Pvalue above 0.05$)$. The position of the anchors had statistically insignificant clinical results (Pvalue above 0.05 ) before, at six month and one year post-operation. Also the number of anchors had statistically insignificant relationship with the UCLA scores at six month and one year (Pvalue above 0.05). At one year, there was a statistically significant reversed relationship between patient age and UCLA score, whereas there was no statistically significant relationship between age and UCLA score pre-operatively or at six months (Fig. 3)

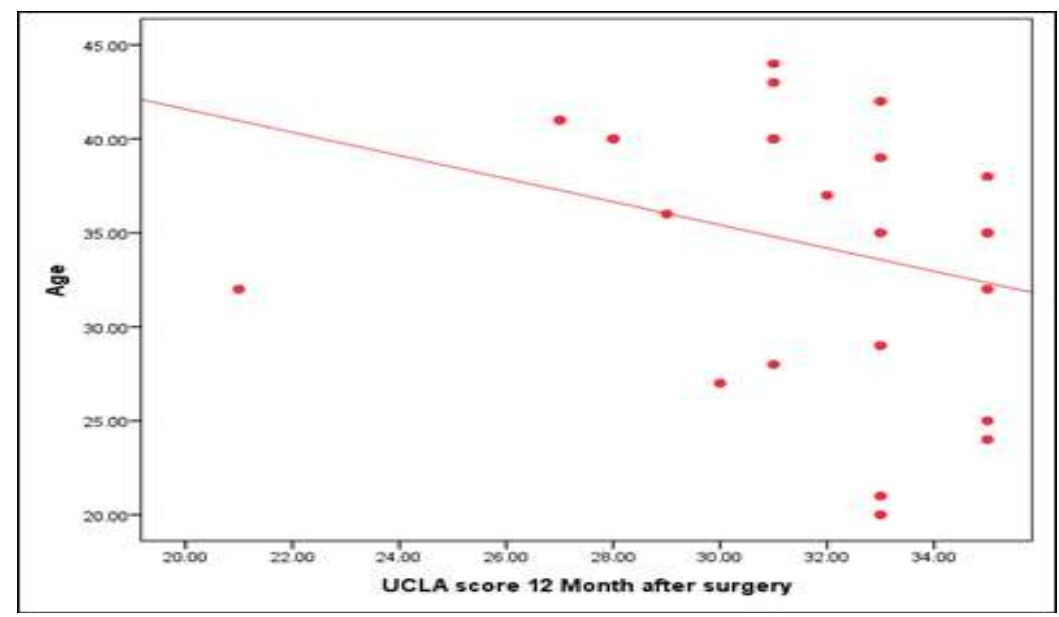

Figure3. The UCLA score 12 month after surgery

Two patients reported higher postoperative disability scores; both had Type 2 SLAP lesions with associated Bankart lesions. Both lesions were repaired arthroscopically in the same time, and they reported higher disability scores due to a sensation of instability without obvious dislocation. They had healed Bankart and SLAP lesions as seen in Magnetic resonance imaging (MRI). Concentrated physiotherapy used for their postoperatively management.

\section{DISCUSSION}

Kim et al. (9) with a Level IV study assessing the use of suture anchors in 34 patients the outcome with repair of isolated type II SLAP tears by arthroscopy. There mean age was 26 years, and the mean follow-up was $33 \pm 9$ months. Using the UCLA scoring system, there result was 27 patients excellent, 5 patients good, 2 patients fair, and no poor results, with $94 \%$ success rate. The DASH score mean improved from 33.2 to 13.6. In addition, they had an excellent to good outcome in all patients with non-overhead athlete though patients in the overhead athlete sports had excellent to good outcome $(89 \%)$. They had $14(41 \%)$ of the 34 patients had choice to go back to their full level of action.

Ide et al. (10) studied the result of type II SLAP repair using anchors suture in overheadthrowing athlete in a Level IV case series. Their mean age was 24 years and their mean followup was 41 months by independent examiner. Excellent in 30 patients, good in 6, fair in 4, 
and, converting into a success rate of $90 \%$ according to modified Rowe scoring system. 30 (75\%) of their patients had the option to come back to their full level of execution without disablement.

Frei et al. (11) in a review investigation of 48 patients who experienced arthroscopic SLAP repair utilizing bioabsorbable anchor sutures demonstrated huge improvement in Constant, ASES and VAS. No distinctions were seen between the results of non-competitors, nonoverhead competitors, recreational overhead competitors, and university overhead competitors, recommending repair of SLAP type II is effective autonomous of the patient's job or game.

Reinold et al. (12) in a prospective investigation of $84 \%$ of competitors with an accompanying SLAP repair came back to rivalry with excellent to good results utilizing the Modified Athletic Shoulder Outcome Scale. In our study examination of 25 patients mean age were 35.23 with the range from 20 to 48 years .There mean follows up was two years. UCLA score before activity was 18.23. After activity UCLA score became 33.24. The UCLA score was excellent in 22 patients, good in two patients and one fair. The patient satisfaction rate was $96 \%$.

What's more, there was a factually critical improvement of the UCLA score somewhere in the range of six month and a year postoperatively. These findings suggest, as do most other studies, that physiotherapy for SLAP repair takes longer than for most other shoulder injuries, and that returning to pre-injury range of motion can take up to a year. This was confirmed by Neri et al. (13) who institute an interim to come back to play about ten months' post SLAP repair in a cohort study performed on 31 competitors. This study discovered a statistically significant negative relationship between the mean age of the patients studied and their UCLA score after one year, but a statistically insignificant relationship between age and UCLA score before surgery or after six months. This could be because the younger was motivated to succeed to return to sports or work.

Denard et al. (14) in their prospective study, Level IV series of 55 patients were presented A good or excellent functional result can be estimated in 87 percent of cases after arthroscopic repair of type II SLAP injuries after a follow-up of 77 months. They have a poor result with workers who are over 40 years old. Studying the results of SLAP repair through age, Neri et al. (15) and Alpert et al. (16) Despite the fact that older aged patients performed slightly inferior than younger, there was no statistical difference in outcome scores as the patient's age increased. However, they discovered that older patients require more time to recover and are more predisposed to complications, particularly stiffness, necessitating early supervision of physiotherapy.

In our study, there were no biomechanical testing implemented, but clinical result displayed statistically insignificant differences between one or two anchor fixation. Similarly it showed insignificant clinical results with anchors that aren't in the middle when compared to central anchors. In Coleman et al. (17) explored the consequences of a Level IV review case arrangement that thought about the result of type II SLAP repair either with or without associated acromioplasty. The study discussed follow-up in 50 of 73 patients who experienced sort II SLAP repair. The two groups SLAP tears were balanced out with a bioabsorbable anchors. At development, the patients finished an L'Insalata questionnaire, a physical assessment, and American Shoulder and Elbow Surgery (ASES) survey were controlled by an independent inspector. As each of the scoring systems, there was statistically insignificant difference in result between these two groups after about 3.5 years follow up. In the study, 47 of the 50 patients (94 percent) were capable to coming back to their pre-injury level of competition, according to the authors.

The retrospective cohort study level III of Enad (18) to compare the result of SLAP repair between group I (isolated SLAP injuries) and group II (associated pathologies as sub acromial impingement and/or partial rotator cuff tear). Their mean age was 31.6 years with a minimum follow-up of 24 months. Bio-absorbable suture anchors were used for arthroscopically repaired SLAP lesions. There was insignificant difference in UCLA scores between these two groups, with $92 \%$ success rate. But, there was a statistically significant alteration in the mean ASES and Visual Analogue Scale (VAS) scores. Group II had a lesser VAS score and a greater ASES score when compared to group I.

In this study we had 15 patients had concomitant impingement problem and in arrangement as the outcomes of Coleman et al. (17) the results of our study showed statistically 
insignificant variance in the UCLA score between one and two years post-operatively. These results, yet, were un-coordinate with further criteria such as the age, traumatic versus non-traumatic onset and, level of activity. In addition, an independent examiner was not known. Therefore, these factors may signal bias, which could lead to erroneous conclusions.

The clinical results of isolated SLAP lesions without concomitant Bankart lesions were studied by numerous researchers. Rhee et al. (19) stated comparable results in their review of arthroscopic procedure of 44 unstable SLAP lesions. $86 \%$ had excellent or good, $14 \%$ fair or poor results according to UCLA scores. Samani et al. (20) also stated that their $88 \%$ success rate with 23 patients, after arthroscopic management, They can resume their pre-injury level of athletic participation. Morgan et al. (21) stated that after one follow-up of 102 arthroscopic procedures of Type 2 lesions that the clinical results $85(83 \%)$ patients were excellent, 14 patients $(14 \%)$ good, and three (3\%) patients fair and there were no poor results according to the UCLA score. Lim et al. (22) stated that the $63 \%$ of the patients with isolated lesions displaying significant improvement in their postoperative disability score. We had, significant improvement in postoperative UCLA score in $(90 \%)$ of the patients with isolated lesions, and they returned to their pre-injury level of activity participation following arthroscopic management.

In this our study, 15 patients (of the 25) had a concomitant impingement problem, $80 \%$ of these patients had associated lesions had improvement in the postoperative UCLA score. Lim et al. (22) reported that $73 \%$ of patients with associated lesions had improvement in the postoperative disability score. This outcome supports the recommendation that SLAP and concomitant lesions should be managed in the same operative session.

\section{CONCLUSION}

Arthroscopic repair of anterior to posterior type II SLAP lesions provides good and improved functional outcome particularly with a component rotator cuff tear is present. There were insignificant clinical results of the patients managed by one or two anchor sutures or the position of the anchors but this need more biomechanical studies for the use of one or two anchor sutures needed and also the position of the anchor.

\section{REFERENCES}

[1] Andrews JR, Carson WG Jr, McLeod WD (1985) Glenoid labrum tears related to the long head of the biceps. Am J Sports Med 13:337341.

[2] Snyder SJ, Karzel RP, Del Pizzo W, Ferkel RD, Friedman MJ, et al.: SLAP lesions of the shoulder. Arthroscopy;1990; 6(4): 274-279.

[3] Silberberg JM, Moya-Angeler J, Martín E, Leyes $\mathbf{M}$ and Forriol F: Vertical versus Horizontal Suture Configuration for the Repair of Isolated Type II SLAP Lesion through a Single Anterior Portal: A Randomized Controlled Trial. Arthroscopy; 2011; 27: 16051613.

[4] Huri G, Hyun YS, Garbis NG and McFarland EG,: Treatment of superior labrum anterior posterior lesions: a literature review ActaOrthopTraumatolTurc; 2014;48: 290-297.

[5] Keener JD and Brophy RH 2009; Superior Labral Tears of the Shoulder: Pathogenesis, Evaluation, and Treatment. J Am AcadOrthopSurg 2009; 17: 627-637.

[6] Mihata T, McGarry MH, Tibone JE, Fitzpatrick MJ, Kinoshita M, and Lee TQ: Biomechanical assessment of type II superior labral anteriorposterior (SLAP) lesions associated with anterior shoulder capsular laxity as seen in throwers: A cadaveric study. Am J Sports Med; 2008;36: 1604-1610.

[7] Bedi A and Allen AA: Superior Labral Lesions Anterior to Posterior Evaluation and Arthroscopic Management. Clin Sports Med 2008;27: 607-630.

[8] Waterman BR, Cameron KL, Hsiao M, Langston JR, Clark NJand Owens BD: Trends in the diagnosis of SLAP lesions in the US military. Knee Surg Sports. TraumatolArthrosc; 2013;37: 22-28.

[9] Kim SH, Ha KI, Kim SH, Choi HJ: Results of arthroscopic treatment of superior labral lesions. J Bone Joint Surg Am; 2002; 84(6): 981-985.

[10] Ide J, Maeda S, Takagi K: Sports activity after arthroscopic superior labral repair using suture anchors in overhead-throwing athletes. Am J Sports Med;2005; 33(4): 507-514.

[11] Friel NA, Karas V, Slabaugh MA, Cole BJ: Outcomes of type II superior labrum, anterior to posterior (SLAP) repair: prospective evaluation at a minimum two-year follow-up. $\mathbf{J}$ Shoulder Elbow Surg;2010; 19(6): 859-867.

[12] Reinold MM, Wilk KE, Hooks TR, Dugas JR, Andrews JR: Thermal-assisted capsular 
shrinkage of the glenohumeral joint in overhead athletes: a 15- to 47-month follow-up. J Orthop Sports PhysTher; 2003; 33(8): 455-467.

[13] Neri BR, El Attrache NS, Owsley KC, Mohr K, Yocum LA: Outcome of Type II Superior Labral Anterior Posterior Repairs in Elite Overhead Athletes Am J Sports Med;2011; 39(1): 114-120.

[14] Denard PJ, LädermannAlexandre, Burkhart SS: Long-Term Outcome after Arthroscopic Repair of Type II SLAP Lesions: Results According to Age and Workers' Compensation Status: Arthroscopy; 2012; 28(4): 451-457.

[15] Neri BR, Emily A, Kvitne RS: Isolated Type II Superior Labral Anterior Posterior Lesions: Age-Related Outcome of Arthroscopic Fixation. Am J Sports Med; 2006; 37(5): 937 942.

[16] Alpert JM, Wuerz TH, O’Donnell TF, Caroll $\mathrm{KM}$, Brucker NN, et al: The effect of age on the outcomes of arthroscopic repair of type II superior labral anterior and posterior lesions. Am J Sports Med; 2010; 38(11): 2299-2303.

[17] Coleman SH, Cohen DB, Drakos MC, Allen AA, Williams RJ, et al: Arthroscopic repair of type II superior labral anterior posterior lesions with and without acromioplasty: A clinical analysis of 50 patients. Am J Sports Med; 2007; 35(5): 749-753.

[18] Enad JG, Kurtz CA: Isolated and combined Type II SLAP repairs in a military population. Knee Surg Sports TraumatolArthrosc;2007; 15(11): 1382-1389.

[19] Rhee YG, Lee DH and Lim CT: Unstable isolated SLAP lesion: clinical presentation and outcome of arthroscopic fixation. Arthroscopy; 2005;21: 1099.

[20] Samani JE, Marston SB and Buss DD: Arthroscopic stabilization of type II SLAP lesions using an absorbable tack. Arthroscopy; 2001;17: 19-24.

[21] Morgan CD, Burkhart SS, Palmeri $M$ and Gillespie M,: Type II SLAP lesions: Three subtypes and their relationships to superior instability and rotator cuff tears. Arthroscopy; 1998;14: 553-565.

[22] Lim MH, Chan BK and Ooi Low C: Arthroscopic Management of Superior Labral Anterior and Posterior (SLAP) and Associated Lesions: Clinical Features and Functional Outcome. Ann Acad Med Singapore; 2008;37: 44-48.

Citation: Emad Abdelhadi, Mohamed El Sadek. Arthroscopic Management of Type II Superior Labrum Anterior to Posterior (SLAP) Lesions: Minimum 2-Year Follow Up. ARC Journal of Orthopedics. 2020; 5(2): 1 7. doi:dx.doi.org/10.20431/2456-0588.0502001.

Copyright: (C) 2020 Authors. This is an open-access article distributed under the terms of the Creative Commons Attribution License, which permits unrestricted use, distribution, and reproduction in any medium, provided the original author and source are credited. 